\title{
Impact of heterogeneities on lateral penumbra in uniform scanning proton therapy
}

\author{
Suresh Rana, Hardev Singh \\ Department of Medical Physics, ProCure Proton Therapy Center, Oklahoma City, Oklahoma, USA.
}

Received October 5, 2013; Revised November 10, 2013; Accepted November 15, 2013; Published Online November 22, 2013

\section{Original Article}

\begin{abstract}
Purpose: In the treatment planning of uniform scanning proton therapy, an aperture block is designed for each beam with a margin, which typically includes the lateral penumbra measured in water (homogenous) medium. However, during real proton therapy treatment, protons may pass through tissues of different densities within the patient's body before they are stopped. The main aim of this study was to investigate the dependency of lateral penumbra on low- and high-density heterogeneities placed in the plateau and spread-out Bragg peak (SOBP) regions. Method: The measurements were performed by placing radiographic films at the isocenter (center of SOBP), and each proton beam was delivered with 150 monitor units using standard beam conditions of the institution. Results and Conclusion: The preliminary results from this study showed that the lateral penumbra of uniform scanning proton beams was less sensitive to the inhomogeneities introduced in the protons beam path. The low-density heterogeneity in the plateau region had more impact on the lateral penumbra when compared to the low-density in the SOBP region. In contrast, the placement of high-density heterogeneity (whether in the plateau or SOBP region) produced a very minimal difference. The overall difference in lateral penumbra among different phantoms was within $\pm 1 \mathrm{~mm}$.
\end{abstract}

Keywords: Lateral penumbra; Uniform scanning proton therapy; Inhomogeneity; SOBP

\section{Introduction}

In uniform scanning proton therapy planning, an aperture block is designed for each beam with a margin, which typically includes the width of the lateral penumbra. A sharp lateral penumbra is particularly important for the treatment sites that have organs at risk (OARs) abutting the target volume. Several groups ${ }^{1-5}$ have performed the measurements and Monte Carlo simulation investigating the dependency of lateral penumbra of proton beams on different beam conditions and geometrical configurations, and it was found that lateral penumbra increases with an increase in range (or energy), depth, and air gap between the aperture/range compensator and phantom.

Corresponding author: Suresh Rana; Department Medical Physics, ProCure Proton Therapy Center, 5901 West

Memorial Road, Oklahoma City, Oklahoma, USA.

Email: suresh.rana@gmail.com

Cite this article as:

Rana S, Singh H. Impact of heterogeneities on lateral penumbra in uniform scanning proton therapy. Int J Cancer Ther Oncol 2013; 1(2):01026. DOI: 10.14319/ijcto.0102.6
The commissioning of treatment planning system (TPS) typically includes the measured data that are obtained in a homogenous medium. However, during real proton therapy treatment, protons may pass through tissues of different densities within the patient's body before they are stopped. The presence of heterogeneity along the protons beam path can change the energy spectrum of proton fluence, and this may change the width of lateral penumbra at a given depth as well as cause the degradation of the Bragg peak region. While previous studies have investigated the lateral penumbra of protons in a homogenous medium, the impact of highand low-density heterogeneities on lateral penumbra for uniform scanning proton beams is yet to be investigated. The principal aim of this study was to perform the experimental measurements to investigate the dependency of lateral penumbra on inhomogeneities placed at different locations upstream from the measurement depth.

\section{Methods and Materials}

The measurements in this study were performed for uniform scanning proton beams on an IBA Cyclotron (IBA, Louvain-la-Neuve, Belgium). A detailed description on the uniform scanning proton therapy system has been provided elsewhere. ${ }^{6,7}$ In order to study the impact of heterogeneities 
on lateral penumbra, we manufactured one homogenous phantom and four inhomogeneous phantoms (Figure 1) utilizing solid-water plates (CIRS, Norfolk, VA), air, and poly-vinyl chloride (PVC) tile.
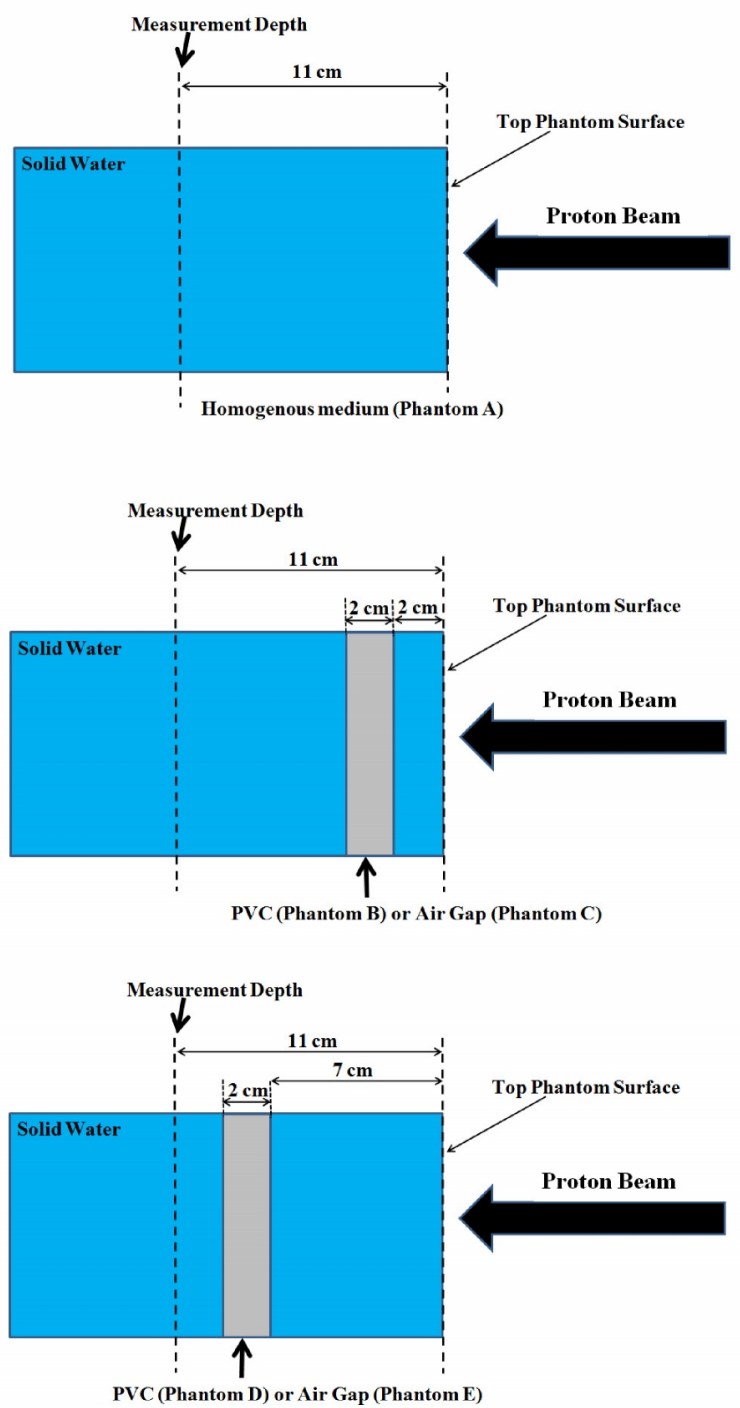

FIG. 1: Schematic diagram of homogenous Phantom $A$ and inhomogeneous Phantoms $B, C, D$, and $E$. In each phantom, the measurement was always taken at $11 \mathrm{~cm}$ depth from the top surface of the phantom. Each phantom had a rectangular area of $30 \times 30 \mathrm{~cm}^{2}$.

\section{Heterogeneities in the plateau region}

An inhomogeneous Phantom $B$ was manufactured by introducing a $2 \mathrm{~cm}$ thick PVC tile at the depth $2 \mathrm{~cm}$ downstream from the top phantom surface as shown in the Figure 1. An inhomogeneous Phantom $C$ was manufactured by replacing the PVC tile in Phantom $B$ with an air gap thickness of $2 \mathrm{~cm}$.

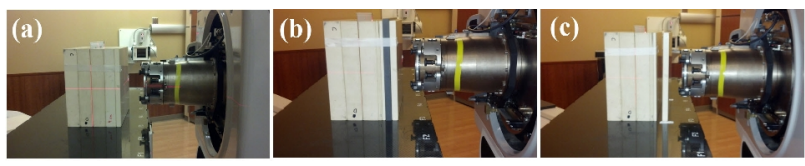

FIG. 2: An experimental setup showing (a) Phantom $A$ (homogenous medium), (b) Phantom $B$ (inhomogeneous medium with $2 \mathrm{~cm}$ thick PVC tile in the plateau region), and (c) Phantom $C$ (inhomogeneous medium with $2 \mathrm{~cm}$ air gap thickness in the plateau region)

\section{Heterogeneities in the spread-out Bragg peak (SOBP) region}

As shown in the Figure 1, an inhomogeneous Phantom $D$ consisted of a $2 \mathrm{~cm}$ thick PVC tile at the depth $7 \mathrm{~cm}$ downstream from the top surface of the phantom. An inhomogeneous Phantom $E$ was created by replacing the PVC tile in Phantom $D$ with an air gap thickness of $2 \mathrm{~cm}$.

\section{Lateral penumbra measurements and analysis}

First, the measurements in each phantom were performed by placing radiographic films (Kodak, Rochester, NY; type EDR-2) at the isocenter, and each proton beam was delivered with 150 monitor units (MU). The measurements were taken for beam conditions listed in Table 1.

TABLE 1: Beam conditions and geometrical configurations for lateral penumbra.

\begin{tabular}{lc}
\hline \hline Beam conditions & Values \\
\hline Range & $16 \mathrm{~cm}$ \\
Modulation & $10 \mathrm{~cm}$ \\
Air gap & $7 \mathrm{~cm}$ \\
Depth = center of SOBP = isocenter & $11 \mathrm{~cm}$ \\
Range compensator thickness & 0 \\
Aperture diameter (circular) & $10 \mathrm{~cm}$ \\
Snout & 10 \\
\hline \hline
\end{tabular}

Abbreviations: SOBP = Spread-out Bragg Peak; Snout10 = maximum field size of $10 \mathrm{~cm}$ circular diameter at the isocenter plane.

(Note: The measurement depth was always at the center of SOBP, which coincided with the isocenter. The proton beam was calibrated to deliver 1cGy absorbed proton dose to water per $M U$ at the center of $S O B P$ under reference conditions (range $=16 \mathrm{~cm}$, modulation $=10 \mathrm{~cm}$, aperture $=10 \mathrm{~cm}$, and air gap $=7 \mathrm{~cm}$ ).

Second, the Medical Film Processor (Konica Minolta Medical \& Graphic, Inc., Tokyo, Japan; model SRX-101A) was used to develop the irradiated EDR-2 films. Third, the Vidar Scanner (Vidar Systems Corporation, Herndon, VA; model DosimetryPro Advantage) was used to scan the processed EDR-2 films. The OmniPro-I'mRT software, version 1.6.009 (IBA Dosimetry, Schwarzenbruck, Germany) was then used for film analysis in order to obtain the measured lateral penumbra $(80 \%-20 \%$ distance). 


\section{Results and Discussion}

Table 2 shows the measured lateral penumbra of uniform scanning proton beams in Phantoms $A, B, C, D$, and $E$. The results indicate that the lateral penumbra of $\mathrm{X}$-magnet is always greater by 0.5 to $0.9 \mathrm{~mm}$ than that of $\mathrm{Y}$-magnet. This was mainly due to the positioning of two scanning magnets in beam line with respect to the virtual source. Specifically, the $\mathrm{X}$-magnet has a virtual source to isocenter distance (SAD) of $220 \mathrm{~cm}$, whereas the Y-magnet has a SAD of 183 $\mathrm{cm}$. Since the geometric penumbra becomes larger with an increase in $\mathrm{SAD}$, the $\mathrm{X}$-magnet with a larger SAD will have a wider geometric penumbra resulting increase in overall lateral penumbra when compared to the lateral penumbra of the Y-magnet with a smaller SAD.

Table 2: Measured lateral penumbra of uniform scanning proton beams in homogenous and inhomogeneous phantoms. (Range $=16$ $\mathrm{cm}$, Modulation $=10 \mathrm{~cm}$, Aperture $=10 \mathrm{~cm}$ Circular Diameter, Measurement Depth $=$ Center of $S O B P=$ Isocenter $)$

\begin{tabular}{lccccc}
\hline \hline & \multicolumn{5}{c}{ Phantoms } \\
\cline { 2 - 6 } & $\begin{array}{c}\mathrm{A} \\
(\mathrm{mm})\end{array}$ & $\begin{array}{c}\mathrm{B} \\
(\mathrm{mm})\end{array}$ & $\begin{array}{c}\mathrm{C} \\
(\mathrm{mm})\end{array}$ & $\begin{array}{c}\mathrm{D} \\
(\mathrm{mm})\end{array}$ & $\begin{array}{c}\mathrm{E} \\
(\mathrm{mm})\end{array}$ \\
\hline X-magnet & 4.2 & 4.5 & 3.8 & 4.5 & 4.1 \\
Y-magnet & 3.6 & 4.0 & 2.9 & 3.7 & 3.5 \\
Average & 3.9 & 4.2 & 3.3 & 4.1 & 3.8 \\
\hline \hline
\end{tabular}

Abbreviations: X-magnet $=$ horizontal scanning magnet, $\mathrm{Y}$-magnet $=$ vertical scanning magnet.

(Note: Phantom A has a homogenous (solid-water) medium, Phantom $B$ has a PVC tile in the plateau region, Phantom Chas an air gap in the plateau region, Phantom D has a PVC tile in the SOBP region, and Phantoms $E$ has an air gap in the SOBP region)

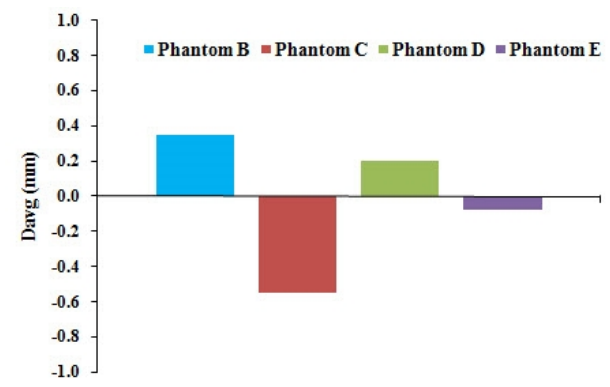

FIG. 3: Comparison of average lateral penumbra results in inhomogeneous phantoms with respect to average lateral penumbra in reference (homogenous) phantom A.

$$
D_{\text {avg }}=\frac{\left(D_{x}+D_{y}\right)}{2}
$$

where, $D_{x}=$ difference in lateral penumbra of $\mathrm{X}$-magnet between inhomogeneous phantoms (B, C, D, and E) and reference phantom $A$, and $D_{y}=$ difference in lateral penumbra of $\mathrm{Y}$-magnet between inhomogeneous phantoms (B, C, D, and E) and reference Phantom A.

\section{Heterogeneities in the plateau region}

The lateral penumbra in Phantom $B$ was greater than that of Phantom $A$ by an average difference of $0.3 \mathrm{~mm}$. In contrast, the results in Phantom $C$ are smaller by an average difference of $0.6 \mathrm{~mm}$ when compared to the ones in Phantom $A$. (Figure 3)

\section{Heterogeneities in the SOBP region}

In comparison to the Phantom $A$, the lateral penumbra in Phantom $D$ was greater (average difference $0.2 \mathrm{~mm}$ ), whereas the lateral penumbra in Phantom $E$ was slightly smaller (average difference $0.1 \mathrm{~mm}$ ). (Figure 3)

The results from this study demonstrated that, at a fixed depth, the high-density heterogeneity can increase the lateral penumbra, whereas the low-density heterogeneity will typically decrease the lateral penumbra. This is mainly due to change in range (or energy) of proton beam as range is dependent on the inhomogeneities introduced in the protons beam path. Additionally, the low-density heterogeneity in the plateau region (Phantom $C$ ) had more impact on the lateral penumbra when compared to the low-density in the SOBP region (Phantom E). (Figure 3 ) In contrast, the placement of high-density heterogeneity (whether in the plateau or SOBP region) produced a very minimal difference.

In this study, we investigated the impact of heterogeneities that have rectangular shape, and each inhomogeneous phantom contained either an air gap or a PVC tile. However, in the real clinical situations, proton beams may pass through multiple non-rectangular heterogeneities with various thicknesses before reaching the target. Although the average difference in lateral penumbra due to inhomogeneities is within $\pm 1 \mathrm{~mm}$, the difference may increase for inhomogeneities with thickness more than $2 \mathrm{~cm}$.

Additionally, if the treatment setup has a proton beam passing through the immobilization devices, one should make an effort in reducing the air gap between such devices and the patient body surface. Since the beam aperture margins are typically selected based on the lateral penumbra of a proton beam for a specific range, it is imperative to investigate the accuracy of treatment planning system in predicting the range of proton beam in the presence of inhomogeneities. An inaccurate prediction of proton range by the treatment planning system will lead to wrong selection of the lateral penumbra, and the aperture margins based on the inaccurate lateral penumbra may lead to over-dose to the normal tissues or under-dose to the target volume during the real clinical treatment. 


\section{Conclusion}

The preliminary results from this study showed that the lateral penumbra of uniform scanning proton beams was less sensitive to the inhomogeneities introduced in the protons beam path. In general, the high-density heterogeneity tend to increase the lateral penumbra, whereas the low-density heterogeneity slightly decreased the lateral penumbra when compared to the results in the homogenous medium. The overall difference in lateral penumbra among different phantoms in this study was within $\pm 1 \mathrm{~mm}$.

\section{References}

1. Urie MM, Sisterson JM, Koehler AM, Goitein M, Zoesman J. Proton beam penumbra: effects of separation between patient and beam modifying devices. Med Phys 1986; 13:734-41.

2. Gottschalk B. Multileaf collimators, air gap, lateral penumbra, and range compensation in proton radiotherapy. Med Phys 2011; 38: i-ii.

3. Oozeer R, Mazal A, Rosenwald JC, Belshi R, Nauraye C, Ferrand R, Biensan S. A model for the lateral penumbra in water of a $200-\mathrm{MeV}$ proton beam devoted toclinical applications. Med Phys 1997; 24:1599-604.

4. Safai S, Bortfeld T, Engelsman M. Comparison between the lateral penumbra of a collimated double-scattered beam and uncollimated scanning beam in proton radiotherapy. Phys Med Biol 2008; 53:1729-50.

5. Rana S, Zeidan O, Ramirez E, Rains M, Gao J, Zheng Y. Measurements of lateral penumbra for uniform scanning proton beams under various beam delivery conditions and comparison to the XiO treatment planning system. Med Phys 2013; 40:091708.

\section{Conflict of interest}

The authors declare that they have no conflicts of interest. The authors alone are responsible for the content and writing of the paper.

6. Zheng Y, Ramirez E, Mascia A, Ding X, Okoth B, Zeidan O, Hsi W, Harris B, Schreuder AN, Keole S. Commissioning of output factors for uniform scanning proton beams. Med Phys 2011; 38:2299-306.

7. Zheng Y, Liu Y, Zeidan O, Schreuder AN, Keole S. Measurements of neutron dose equivalent for a proton therapy center using uniform scanning proton beams. Med Phys 2012; 39:3484-92. 